\title{
Transcranial direct current stimulation of the frontal eye fields during pro- and antisaccade tasks
}

\section{Ryota Kanai *, Neil Muggleton and Vincent Walsh}

Department of Psychology, Institute of Cognitive Neuroscience, University College London, London, UK.

\section{Edited by:}

Andre R. Brunoni, Universidade de São Paulo, Brazil

\section{Reviewed by:}

Andrea Antal, University Medical Center Goettingen, Germany

Thiago Leiros Costa, Universidade de São Paulo, Brazil

\section{*Correspondence:}

Ryota Kanai, Institute of Cognitive Neuroscience, University College London, 17 Queen Square, WC1N 3AR London, UK.

e-mail: kanair@gmail.com
Transcranial direct current stimulation (tDCS) has been successfully applied to cortical areas such as the motor cortex and visual cortex. In the present study, we examined whether tDCS can reach and selectively modulate the excitability of the frontal eye field (FEF). In order to assess potential effects of tDCS, we measured saccade latency, landing point, and its variability in a simple prosaccade task and in an antisaccade task. In the prosaccade task, we found that anodal tDCS shortened the latency of saccades to a contralateral visual cue. However, cathodal tDCS did not show a significant modulation of saccade latency. In the antisaccade task, on the other hand, we found that the latency for ipisilateral antisaccades was prolonged during the stimulation, whereas anodal stimulation did not modulate the latency of antisaccades. In addition, anodal tDCS reduced the erroneous saccades toward the contralateral visual cue. These results in the antisaccade task suggest that tDCS modulates the function of FEF to suppress reflexive saccades to the contralateral visual cue. Both in the prosaccade and antisaccade tasks, we did not find any effect of tDCS on saccade landing point or its variability. Our present study is the first to show effects of tDCS over FEF and opens the possibility of applying tDCS for studying the functions of FEF in oculomotor and attentional performance.

Keywords: saccade, antisaccade, frontal eyefield, transcranial direct current stimulation

\section{INTRODUCTION}

Delivering direct current from scalp to cortex over several minutes has been shown to induce a long-lasting change in cortical excitability. This stimulation technique called transcranial direct current stimulation (tDCS) has been applied to many cortical areas, most notably the motor cortex (Priori et al., 1998; Nitsche and Paulus, 2000, 2001; Baudewig et al., 2001) and visual cortical areas (Antal et al., 2003, 2004, 2006). In tDCS, anodal stimulation generally enhances cortical excitability and cathodal stimulation suppresses it. TDCS is believed to interfere with brain functions by modulating the spontaneous firing rate of the cortex under the stimulating electrode by depolarizing the membrane potential with anodal stimulation or by hyperpolarizing it with cathodal stimulation (Bindman et al., 1962; Creutzfeldt et al., 1962; Fregni and Pascual-Leone, 2007). For example, motor-evoked potentials (MEP) in response to transcranial magnetic stimulation (TMS) over the motor cortex become larger after anodal stimulation, while they are reduced following cathodal stimulation (Nitsche and Paulus, 2000, 2001). An analogous study on the visual cortex showed a similar pattern of results: Antal et al. (2003) measured excitability of the visual cortex by measuring the TMS-induced phosphene threshold over the occipital cortex following anodal or cathodal stimulation. Consistent with the results for the motor cortex, the phosphene threshold decreased after anodal stimulation and increased after cathodal stimulation.

Abbreviations: BOLD, blood-oxygen-level dependent; FEF, frontal eye field; MEP, motor-evoked potential; rTMS, repetitive TMS; tDCS, transcranial direct current stimulation; TMS, transcranial magnetic stimulation.
The goal of the present study was to examine whether tDCS can reach and modulate the excitability of the frontal eye fields (FEF), which is one of the key areas involved in controlling eye movements and selective attention (Robinson and Fuchs, 1969; Mohler et al., 1973; Wurtz and Mohler, 1976; Schall and Thompson, 1999; Serences and Yantis, 2007). Since no study has targeted FEF with tDCS to date, our primary goal was to observe possible effects of tDCS over FEF. Toward this goal, our first set of experiments examined how tDCS over the FEF modulates saccade properties in a simple prosaccade task in which subjects are required to make an eye movement toward a peripheral stimulus. The FEF in each hemisphere is known to be involved in the control of saccades in the contralateral direction. Since saccade latency is known to be dependent on the time when the firing rate of FEF reaches a threshold for saccade execution (Hanes and Schall, 1996), it was expected that the firing rate in FEF would reach the threshold for saccade execution more quickly if the baseline firing rate was increased by anodal tDCS. Conversely, suppression of the firing rate in FEF was expected to prolong the saccade latency. Alternatively, saccade latency can be modulated by changes in the threshold for saccade generation (Reddi et al., 2003). Thus, it is also conceivable that tDCS modulates saccade latency by changing the threshold rather than changing the baseline activity level.

There are several lines of evidence that impairment or inactivation of the FEF prolongs saccade latency in the prosaccade task. Patients with lesions in the FEF show a prolonged latency for prosaccades toward the direction contralateral to the lesion in an overlap condition in which the initial fixation point remained displayed even after the saccade target appeared (Gaymard et al., 
1999). In monkeys, acute inactivation of the FEF leads to a prolongation of saccade latency in the direction contralateral to the inactivated FEF (Sommer and Tehovnik, 1997; Dias and Segraves, 1999). Small prolongation of ipsilateral saccades was also reported, but this effect was much smaller than the effects on contralateral saccades. Previous TMS studies show that the saccade latency in the prosaccade task is increased following $10 \mathrm{~min}$ of offline $1 \mathrm{~Hz}$ repetitive TMS (rTMS) or theta burst stimulation over FEF (Nyffeler et al., 2006a,b). These studies together suggest that activity in FEF influences the latency of prosaccades.

In the second part of our study, we examined possible effects of tDCS over FEF in the antisaccade task (Hallet, 1978) in which subjects are required to suppress reflexive eye movement to a visual cue and generate a volitional saccade to the location opposite to the cue (for reviews, see Everling and Fischer, 1998; Munoz and Everling, 2004). FEF has been thought to play an important role in producing successful antisaccades. Earlier neuroimaging studies show that the activation of FEF is greater during blocks of antisaccade trials as compared to blocks of prosaccade trials or fixation (O’Driscoll et al., 1995; Müri et al., 1998; McDowell et al., 2002; Matsuda et al., 2004).

One important role of FEF in the antisaccade task is the generation of volitional saccades. Patients with a localized lesion in FEF show a normal percentage of errors in suppressing reflexive saccades, whereas the latency of correct antisaccades was increased bilaterally (Pierrot-Deseilligny et al., 1991; Rivaud et al., 1994; Gaymard et al., 1999). Neuroimaging studies using an event-related design showed that the activity of FEF is strongly associated with the generation of a volitional saccade (Ettinger et al., 2008). On the other hand, FEF is also implicated in the suppression of reflexive saccades (Hunt et al., 2004). Patients with lesions to FEF and possibly other frontal areas, including the dorsolateral prefrontal cortex, have difficulty in suppressing reflexive saccades in the antisaccade task (Guitton et al., 1985). Neuroimaging studies in which the prosaccade and antisaccade trials were randomly assigned by a visual cue showed differential BOLD activity in FEF even before the appearance of the saccade target. Therefore, the enhanced activity in the FEF after the instruction for an antisaccade was interpreted as reflecting the preparatory set for suppressing a response to the upcoming saccade target (Connolly et al., 2002; Cornelissen et al., 2002). We discuss our results of tDCS over FEF in the antisaccade task in the context of these two FEF functions, namely, generation of volitional saccades and suppression of reflexive saccades.

\section{MATERIALS AND METHODS \\ PROSACCADE TASK WITH BILATERAL tDCS \\ Subjects}

Sixteen subjects ( 6 males and 10 females) were recruited from the community of University College London. They were all righthanded and had normal or corrected-to-normal visual acuity. Informed written consent was obtained from each participant. During the experiment, they sat $57 \mathrm{~cm}$ away from a $17^{\prime \prime}$ CRT monitor running with a $100-\mathrm{Hz}$ refresh rate. Their head was immobilized by a chin- and headrest on which an eyetracker (Eyelink, SMI, Berlin/Germany) was mounted. The experiment conformed to the Declaration of Helsinki and was approved by a local (University College London) ethical committee. Informed consent was obtained from all subjects.

\section{Transcranial direct current stimulation}

Direct current was delivered by a constant current stimulator (Schneider Electronics, Gleichen, Germany) via a pair of anodal and cathodal electrodes placed over left and right FEF. The electrodes were covered with flat synthetic sponges soaked in $0.9 \%$ $\mathrm{NaCl}$ solution. The contact surface of the sponges was $3 \mathrm{~cm} \times 3 \mathrm{~cm}$ for both electrodes. The maximum current density under the electrode was $0.11 \mathrm{~mA} / \mathrm{cm}^{2}$. This is slightly higher than typical current density ranging 0.029 and $0.08 \mathrm{~mA} / \mathrm{cm}^{2}$ (Nitsche et al., 2008) and was chosen in order to increase the focality and effectiveness of the stimulation (i.e., the area of the electrode was lower than that typically used). The electrodes were fixed to the target positions (see below) with rubber bands. The intensity of tDCS was slowly ramped up to $500 \mu \mathrm{A}$ over the initial $10 \mathrm{~s}$ of stimulation. If participants felt comfortable with the stimulation, we further increased the intensity up to $1000 \mu \mathrm{A}$ and continued the stimulation for $10 \mathrm{~min}$. Typically the voltage required to achieve $1000 \mu \mathrm{A}$ stimulation without unpleasant sensations was below $20 \mathrm{~V}$. If participants reported any unpleasant sensation at the scalp under the electrodes (usually the anodal electrode), we added saline to the sponge and waited until the impedance between the electrodes decreased.

\section{Localization of FEF}

Structural MRI images of all participants were obtained prior to the experiment. The positions of left FEF and right FEF were determined for individual participants using FSL (FMRIB Software Library, Oxford, UK) in the following transformation steps; first we converted individual structural images to the standard MNI coordinates, and then the coordinates for left FEF and right FEF in MNI coordinates were transformed back to the real image space. The MNI coordinates for L-FEF and R-FEF were $(-32.3$, $-4.4,49.8)$ and $(31.3,-4.5,50.9)$. Those values are calculated from the Talairach coordinate given in Paus (1996) by converting the coordinates from Talairach space to MNI space. The individual specific target sites were used to guide frameless stereotaxy using the Brainsight system (Rogue Research, Montreal, Canada).

While it is known that the exact location of FEF can be variable between individuals, our approach of targeting FEF using the standard coordinate has proved to be sufficiently effective for interfering with functions of FEF (e.g., Muggleton et al., 2003; Juan et al., 2008; Nuding et al., 2009; Bardi et al., 2012). A recent study compared three different types of neuronavigation methods by comparing effects of TMS over the parietal cortex using individual fMRI results, the standard coordinate and the P4 of the international 10-20 system (Sack et al., 2009). The study showed that the fMRI-based neuronavigation is more effective, but the standard coordinate was also effective if about twice as many subjects were tested. On the other hand, neuronavigation based on the 10-20 system required many more subjects (about 10 fold more) to reach comparable statistical power. Thus, given the number of subjects in each of our experiment ( $n=16$ for each experiment), we expected that a coordinate based approach without fMRI validation would be sufficient for our purpose. Moreover, the electric current delivered through the scalp spreads very rapidly and the 
electrode sponges cover a large area $(3 \mathrm{~cm} \times 3 \mathrm{~m})$ relative to TMS. We chose the coordinate based approach to target FEF, because we did not expect precise localization of FEF for individual subjects separately would improve the effectiveness of tDCS due to the relatively large electrodes and volume conduction of the current used in this technique.

\section{Design of the experiment}

The task for the participants was to make an eye movement to a target (black square) in response to the onset of the target presented in one of two designated positions $\left(8.1^{\circ}\right.$ away from the fixation horizontally), which were indicated by hollow square frames (Figure 1A). The target position (left or right) was randomized across trials, and the participants completed 40 trials in one block. Each block was completed within 5 min including calibration of the eye tracker at the beginning of each block.

The central fixation point remained on the screen even after the saccade target appeared. In a study of FEF patients, no impairment was found in a gap condition in which the initial fixation point was removed $200 \mathrm{~ms}$ before the onset of the saccade target (Pierrot-Deseilligny et al., 1991; Rivaud et al., 1994). In order to observe possible effects of tDCS over the FEF, we therefore used the overlap paradigm for the prosaccade experiment as described above.

First, each participant completed one practice block of 40 trials before starting the actual experimental session. After the practice, participants completed a session consisting of five pairs of blocks (10 blocks in total; see Figure 1B). The first pair of blocks (Blocks 1 and 2) were the baseline condition to which subsequent performance was compared. TDCS was delivered during the second pair of blocks (Blocks 3 and 4) for $10 \mathrm{~min}$. The third, fourth, and fifth
A

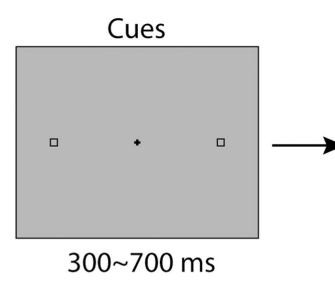

B

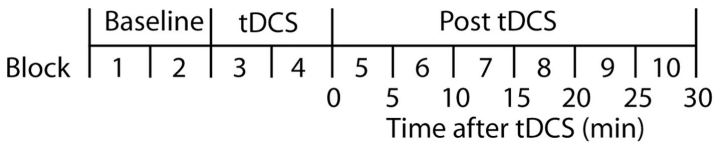

FIGURE 1 | Experimental design. (A) Stimulus display for a single trial is illustrated. Two boxes were displayed continuously throughout a trial. A black square appeared within one of the boxes after a variable delay $(300-700 \mathrm{~ms})$. In the prosaccade experiments, subjects were asked to make direct their gaze to the visual target as quickly and accurately as possible. In the antisaccade task, they were asked to make an eye movement away from the visual cue, directing to the square on the opposite side. (B) The time course of an experimental session. A session started with two blocks of baseline conditions before tDCS, followed by another two blocks with tDCS. After the tDCS blocks, six more blocks were completed to observe the time course of possible effects of tDCS. Each block duration was adjusted to be 5 min including the calibration of the eye tracker and a brief rest between blocks. pairs were used to estimate long-lasting effects of tDCS on saccade properties during 0-10, 10-20, and 20-30 min following the termination of tDCS, respectively. At the beginning of each block, the eye tracker was calibrated and drift correction was performed at the beginning of each trial.

For half of the participants, the anodal electrode was placed over left FEF and cathodal over right FEF, and the polarity of the electrodes was reversed for the other half of the participants. Saccade data were analyzed according to whether saccade direction was contralateral to the anode or the cathode.

\section{Eye movement recording and analysis}

The gaze direction of the right eye was sampled at $250 \mathrm{~Hz}$ using EyeLink system (SR Research Ltd, Ontario, Canada). We calculated saccade latency, landing position with respect to the target and variability of saccade amplitudes. To determine the onset and offset of a saccade, we used a velocity criterion. When eye velocity exceeded $26.8^{\circ} \mathrm{s}$, that time point was regarded as the onset of a saccade and when eye velocity went down again below 26.8 $\mathrm{deg} / \mathrm{s}$, the time point immediately after this sample was regarded as the termination of the saccade. Data points for saccade latencies, amplitudes, and landing positions were collapsed across two consecutive 5 min sessions $(40$ trials $\times 2)$ in order to increase the robustness of estimating these parameters.

Median rather than mean was used for saccade latency because the distribution of saccade latencies is typically skewed (e.g., Carpenter and Williams, 1995). However, the results of statistical analysis showed qualitatively identical patterns when the mean saccade latency was used. In the present paper, we report only the results of median based latency estimates.

Trials were rejected from the analyses if eye position at the time of target onset deviated from the fixation more than $1.8^{\circ}$, if the first saccade after target onset was directed to the opposite side from the target, or if saccade latency was longer than $400 \mathrm{~ms}$ or shorter than $50 \mathrm{~ms}$. Trials were sorted according to whether saccade direction was contralateral to anode position or cathode position.

\section{PROSACCADE TASK WITH UNILATERAL tDCS} Subjects

Sixteen new subjects participated in the cathode only condition $(5$ males and 11 females), and yet another 16 subjects ( 8 males and 8 females) participated in the anode only condition. None of them had participated in the bilateral tDCS experiment. The experiment conformed to the Declaration of Helsinki and was approved by a local (University College London) ethical committee. Informed consent was obtained from all subjects.

\section{Design of the experiments}

The experiments were conducted in the same manner as the first experiment. Both for the anodal experiment and cathodal experiment, half of subjects in each experiment had tDCS on the right side and the other half on the left. Data were sorted according to whether saccade target appeared contralateral to the tDCS site.

\section{Transcranial direct current stimulation}

The duration and intensity parameters were identical to the bilateral experiment. When stimulating the motor cortex with tDCS, 
reference electrodes are often placed on the contralateral forehead because other electrode montages were found to be ineffective (Nitsche and Paulus, 2000). However, we avoided using the forehead position for the reference electrode in order to avoid unexpected effects of stimulating the cortical structures under the reference electrode, as comparisons would be made between contralateral and ipisilateral saccades in our experiment. Therefore, the reference was fixed on the shoulder ipsilateral to the stimulation electrode, a montage known to be effective for stimulating the motor cortex (Priori et al., 1998). For the reference electrode, we used a larger electrode $(5 \mathrm{~cm} \times 7 \mathrm{~cm})$ to minimize the current density and thereby sensation on the skin. No sham condition was included in this study.

\section{ANTISACCADE TASK WITH UNILATERAL tDCS Subjects}

As in the prosaccade experiment with unilateral tDCS, 16 subjects participated in the cathode only condition, and another 16 subjects participated in the anode only condition. Three of participants who took part in the cathode condition took part also in the prosaccade experiment (two of them were in the anode condition and one of them was in the cathode condition). Three participants in the anode condition took part also in the prosaccade experiment (two of them were in the anode condition and one of them was in the cathode condition). However, these two experiments involved different tasks and were conducted at least 6 months apart and therefore carry-over and practice effects are expected to be negligible. The experiment conformed to the Declaration of Helsinki and was approved by a local (University College London) ethical committee. Informed consent was obtained from all subjects.

\section{Design of the experiments}

The stimuli were identical to the prosaccade experiments described above. The only difference was that the subjects were instructed to make a saccade to the box opposite to the black cue. Both for the anodal experiment and cathodal experiment, half of subjects in each experiment had tDCS on the right side and the other half on the left. Data were sorted according to whether the correct saccade direction was contralateral or ipsilateral to the tDCS site. The stimulation methods for tDCS were identical to the prosaccade experiment with unilateral tDCS described above. No sham condition was included in this study.

\section{RESULTS \\ BILATERAL tDCS OVER FEF IN THE PROSACCADE TASK}

In the first experiment, we applied tDCS bilaterally with anode over one FEF and cathode over the other. Since anode and cathode often produce opposite effects, we expected this bilateral tDCS configuration would yield a large difference between leftward and rightward saccade properties if any effect was to be observed.

The time course of saccade latency is shown in Figure 2A. In order to estimate effects of tDCS and the time course of any effects, we subtracted the baseline saccade latency obtained before tDCS from the data obtained in the subsequent $\mathrm{tDCS}$ session and post-tDCS sessions (Figure 2D). The shifts of saccade latency from the baseline were analyzed using ANOVA with the polarity of tDCS and time course as factors. We found a main effect of $\mathrm{tDCS}(F(1,45)=9.62, p<0.01)$. Time course was not significant $(F(3,45)<1)$ and there was no interaction between the two factors $F(3,45)<1)$. These results indicate that the latency of saccades in the direction contralateral to anodal tDCS became shorter than that of saccades contralateral to cathodal tDCS. These results suggest that anodal tDCS facilitated contralateral saccade generation and/or cathodal tDCS suppressed contralateral saccade generation.

In order to examine whether other metrics of saccades were altered by tDCS, we used two measures to estimate accuracy of saccades. One was the variability of the saccade landing point, which we calculated as the standard deviation of the horizontal position of the saccade landing point. The other measure was the mean landing position with respect to the target position. These two measures, variability and landing position, are indicative of the level of noise and systematic bias in saccade execution, respectively (e.g., White et al., 1994).

There were no statistically significant effects of anodal or cathodal stimulation on accuracy or variability. For the landing point of saccades, a repeated measures ANOVA with tDCS and time course as factors did not show any significant effect on the landing point ( $\mathrm{tDCS}, F(1,45)<1$; time course, $F(3,45)=1.19$, $p=0.326$; interaction, $F(3,45)=1.71, p=0.178)$. For the variability of saccade amplitude, a repeated measures ANOVA with tDCS and time course as factors did not show any significant effect on the variability $(\mathrm{tDCS}, F(1,45)=2.997, p=0.104$; time course $F(3,45)<1$; interaction, $F(3,45)<1)$.

These results together show that tDCS primarily influences saccade latency but has no effect on saccade amplitude or its variability. The effect on latency was specific to saccades contralateral to the FEF stimulated by the anode, suggesting that anodal stimulation shortened saccade latency. The latency difference produced by tDCS was $7.8 \mathrm{~ms}$ on average across the entire time course of the experiment. However, it is unclear whether the change in saccade latency was due to the anodal stimulation only, or alternatively was caused by a combination of anodal and cathodal effects, which shifted the balance between the left and right FEF.

\section{UNILATERAL tDCS OVER FEF IN THE PROSACCADE TASK}

In order to isolate effects of anodal and cathodal tDCS, we applied either anodal or cathodal tDCS over one FEF with the reference electrode over the ipisilateral shoulder (deltoid muscle).

The results of anodal tDCS are shown in Figure 2B. As in the bilateral experiment, we assessed the effects of tDCS by subtracting the baseline obtained before the tDCS delivery (Figure 2E). Consistent with the bilateral tDCS experiment, anodal stimulation shortened the latency of saccade contralateral to the stimulation site $(F(1,45)=4.70, p<0.05)$. As in the bilateral experiment, we did not find a significant main effect of the time course $(F(3,45)=1.25, p=0.304)$ nor an interaction between time course and saccade direction with respect to the stimulation site $(F(3,45)<1)$. The difference in saccade latency between tDCS and unstimulated sites was $6.4 \mathrm{~ms}$. Neither the landing position nor its variability were affected by tDCS (all $F$-values $<1$ except the effect of tDCS on saccade variability, $F(1,45)=2.07$, $p=0.170)$. 

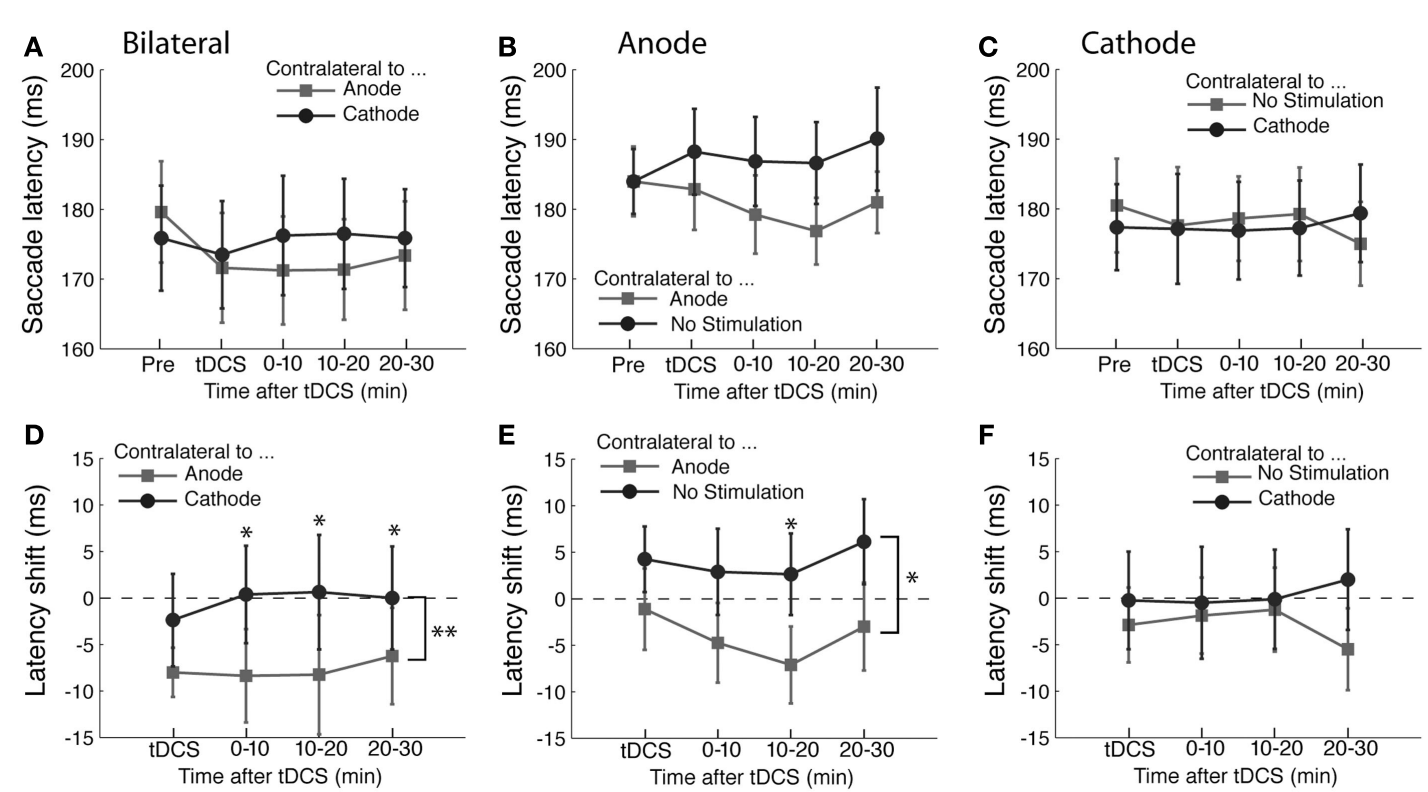

FIGURE 2 | Effects of tDCS in the prosaccade task. (A-C) The mean saccade latency is plotted as a function of the time course for the (A) bilateral, (B) anodal only, and (C) cathodal only conditions. (D-F) Shifts in saccade latency from the baseline condition before tDCS are plotted as a function of time from the beginning of tDCS blocks for saccades. In (A) and (D), the light gray squares represent the latency of saccade contralateral to the anode and the dark gray circles the latency contralateral to the cathode. In (B) and (E), the light gray squares represent the latency of saccade contralateral to the anode and the dark gray circles the latency contralateral to the unstimulated side. In (C) and (F), the light gray squares represent the latency of saccade contralateral to the unstimulated side and the dark gray circles the latency contralateral to the cathode. The error bars correspond to one standard error of the mean (SEM). Asterisks $\left({ }^{*} p<0.05\right.$ and $\left.{ }^{* *} p<0.01\right)$ indicate main effects of saccade direction with respect to stimulation site $(\mathbf{D}, \mathbf{E})$ and the significantly different pairs revealed by a post hoc two-tailed $t$-test test with Bonferroni correction.
The time course of saccade latency in the experiment with cathodal tDCS is shown in Figure 2C. As in the bilateral experiment, we analyzed the change in saccade latency with respect to the baseline obtained before the tDCS delivery (Figure 2F). There was no significant difference in saccade latency between the direction contralateral to tDCS side and the direction contralateral to the unstimulated side $(F(1,45)=1.09, p=0.313)$. Neither was there an effect of time course $(F(3,45)<1)$ nor an interaction between time course and saccade direction with respect to stimulation site $(F(3,45)<1)$. Accuracy measures (i.e., variability and landing point) did not show a significant shift after cathodal tDCS either. The results of ANOVA on variability were non-significant for the effect of saccade direction with respect to the stimulation site $(F(1,45)=2.68, p=0.12)$, time course $(F(3,45)<1)$ and interaction $(F(3,45)=1.24, p=0.31)$. As for the landing point, there were no statistically significant effects of saccade direction $(F(1,45)=1.1, p=0.31)$, time course $(F(3,45)<1)$ or their interaction $(F(3,45)=1.68, p=0.19)$. Therefore, unilateral cathodal stimulation over FEF did not have any effect on saccade properties analyzed in the present study. This suggests that the effect of bilateral tDCS we found in the prosaccade experiment 1 was likely due to the anodal stimulation rather than cathodal stimulation. Thus, a lack of $\mathrm{tDCS}$ effects in unilateral conditions (e.g., cathodal tDCS in the prosaccade experiment) could be attributed to attenuation of tDCS effects rather than genuine absence of effects.

\section{UNILATERAL tDCS OVER FEF IN THE ANTISACCADE TASK}

The results of the antisaccade experiment are shown in Figure 3. The results of anodal tDCS are summarized in Figures 3A,C. Unlike the prosaccade experiments, we did not find any statistically significant effects of anodal tDCS in the antisaccade task. ANOVA on saccade latency showed a main effect of time course $(F(3,45)=3.11, p<0.05)$, but there was no effect of tDCS $(F(1,45)<1)$ or interaction between time and tDCS condition $(F(3,45)<1)$. We did not find systematic effects of anodal tDCS on the variability of saccade amplitudes (Time, $F(3,45)<1$; tDCS, $F(1,45)=1.78, p=0.20$; interaction, $F(3,45)<1)$ nor saccade landing points (Time, $F(3,45)=2.26, p=0.09$; $\mathrm{tDCS}, F(1,45)<1$; interaction, $F(3,45)=1.53, p=0.21)$.

On the other hand, cathodal tDCS had an effect in the antisaccade task (Figures 3B,D). ANOVA on saccade latency revealed a significant interaction between time and tDCS condition $(F(3,45)=3.45, p<0.05)$. This interaction was a result of the difference in latency during tDCS $(T(15)=-4.13, p<0.01)$. This indicates that ipsilateral antisaccades were delayed by cathodal tDCS compared with contralateral antisaccades (Figure 3B). The difference was not significant at any other time points (all $T$-values $<1)$. Both the factors of time course $(F(3,45)<1)$ and saccade direction with respect to stimulation site $(F(1,45)=1.53$, $p=0.24)$ were statistically significant. We did not find any systematic effects of cathodal tDCS on other saccade metrics as the variability of saccade amplitudes (time course, $F(3,45)<1$; 

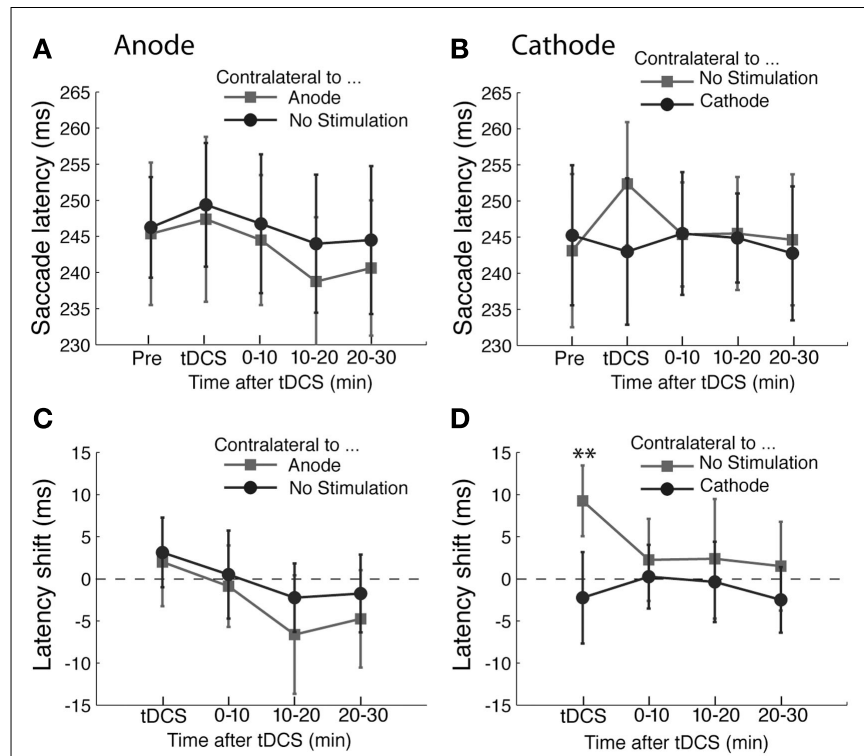

FIGURE 3 | Effects of tDCS in the antisaccade task. (A,B) The mean saccade latency is plotted as a function of the time course for the (A) anodal only and (B) cathodal only conditions. (C,D) Shifts in saccade latency from the baseline condition before tDCS are plotted as a function of time from the beginning of tDCS blocks for saccades. In (A) and (C), the light gray squares represent the latency of saccade contralateral to the anode and the dark gray circles the latency contralateral to the unstimulated side. In (B) and (D), the light gray squares represent the latency of saccade contralateral to the unstimulated side and the dark gray circles the latency contralateral to the cathode. The error bars correspond to one standard error of the mean (S.E.M.). Asterisks ( ${ }^{*} p<0.05$ and ${ }^{* *} p<0.01$ ) indicate main effects of saccade direction with respect to stimulation site (D) and the significantly different pairs revealed by a post hoc two-tailed $t$-test with Bonferroni correction.
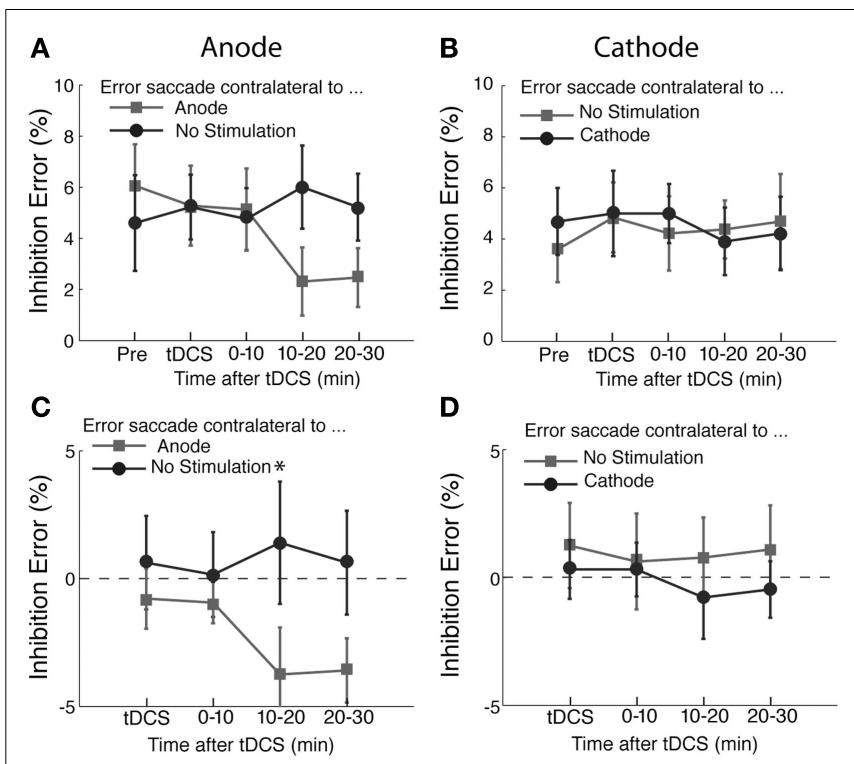

FIGURE 4 | Effects of tDCS on inhibition errors in the antisaccade task. The percentage of inhibition trials in which subjects made an error saccade toward the visual cue. In (A) and (C), the light gray squares represent the percentage of errors of making saccade to the visual cue contralateral to the anode and the dark gray circles the percentage of error saccades to the visual cue contralateral to the unstimulated side. In (C), the data shown in (A) was replotted after subtracting the pre-tDCS baseline to highlight changes induced by tDCS. In (B) and (D), the changes of the error rates from the pre-stimulation blocks are shown in the same format as (A) and (C). In (D), the data shown in (B) was replotted after subtracting the pre-tDCS baseline to highlight changes induced by tDCS. The error bars correspond to one standard error of the mean (SEM). Asterisks $\left({ }^{*} p<0.05\right.$ and ${ }^{*} p<0.01$ ) indicate the significantly different pairs revealed by a post hoc two-tailed $t$-test with Bonferroni correction. saccade direction, $F(1,45)<1$; interaction, $F(3,45)<1)$ or saccade landing points (time course, $F(3,45)<1$; saccade direction, $F(1,45)<1$; interaction, $F(3,45)=2.21, p=0.09)$.

\section{INHIBITION ERRORS IN THE ANTISACCADE TASK AFTER tDCS}

In order to evaluate possible changes in the ability to suppress automatic saccades toward the peripheral cue, we calculated the percentage of trials in which the subjects erroneously made a saccade to the peripheral cue and examined possible changes in the inhibition error rates due to tDCS.

The percentages of inhibition errors are shown in Figure 4. We found an effect of anodal tDCS as shown in Figures 4A,C. There was a significant interaction between the time course and saccade direction with respect to the stimulation site $(F(3,45)=3.05, p<0.05)$. A post hoc test revealed that this interaction was due to a significant difference in the error rate during the period immediately after the delivery of tDCS $(T(15)=-2.35, p<0.05)$. There was no significant effect of time course $(F(3,45)<1)$ or saccade direction $(F(1,45)=3.4$, $p=0.08)$. We did not find a significant effect of cathodal tDCS on the inhibition error rate (Figures 4B,D; Time course, $F(3,45)<1$; saccade direction, $F(1,45)=1.30, p=0.27$; interaction, $F(3,45)<1)$.

\section{DISCUSSION}

In the present study, we have shown that tDCS over FEF can modulate saccade properties in prosaccade and antisaccade tasks. Our main findings are summarized as (1) anodal tDCS shortens the latency of contralateral prosaccades, (2) cathodal tDCS lengthens the latency of ipsilateral antisaccades, (3) anodal tDCS reduces automatic error saccade to the contralateral cue, and (4) saccade amplitudes and their variability are unaffected by tDCS. In this section, we elaborate on each of these points and provide comparisons with related TMS and lesion studies on FEF and saccade generation.

\section{FACILITATION OF REFLEXIVE SACCADE BY ANODAL tDCS}

The only effect we observed by tDCS over FEF in the prosaccade experiment was shortening of saccade latency in the direction contralateral to anodal stimulation. The positive effect of anodal tDCS and no effect of cathodal tDCS support the idea that the effect we found in the bilateral tDCS experiment is primarily due to the shortening of saccade latency due to activation of FEF by anodal stimulation rather than lengthening of latency due to suppression of FEF by cathodal stimulation.

Anodal tDCS is believed to increase the spontaneous firing rate of the cortex under the stimulating electrode by depolarizing the 
membrane potential (Bindman et al., 1962; Creutzfeldt et al., 1962; Fregni and Pascual-Leone, 2007). Saccade latency is known to be dependent on the time when the firing rate of FEF reaches a threshold for saccade execution and the variability of saccade latency is due to the difference in the rate of increase in firing rate across trials (Hanes and Schall, 1996). In line with these findings, our results can be interpreted such that the firing rate in FEF reached the threshold for saccade execution more quickly due to the increased firing rate induced by anodal tDCS. Alternatively, our results are also compatible with the hypothesis that tDCS modulates the threshold for generating saccades and thereby shortening saccade latency. Further studies are needed to determine how tDCS influenced saccade latency. The shortening of the latency continued even up to 30 min after the tDCS delivery (Figures 2A,D). Since we measured the effect of tDCS only up to $30 \mathrm{~min}$ after the delivery of tDCS, it is unclear how long the effect lasts after anodal tDCS.

\section{SLOWING OF IPISILATERAL ANTISACCADE BY CATHODAL TDCS}

In the antisaccade task, cathodal tDCS delayed the generation of antisaccades toward the direction ipisilateral to the stimulation site. This is informative for understanding the functions of FEF and possibly the neighboring areas stimulated by tDCS in the antisaccade task. As we outlined in the introduction, FEF has been implicated in generation of volitional saccades and suppression of reflexive saccades. These two presumed functions of the FEF make distinct predictions as to the consequences of tDCS over FEF during the antisaccade task. If the generation of volitional saccades were to be suppressed by cathodal tDCS over FEF, the latency of contralateral saccades would have been prolonged. However, this is not clearly the case. Instead, the prolongation occurred only for ipisilateral saccades. This result may be regarded as somewhat puzzling, but can be understood if we assume that a successful antisaccade consists of a serial, rather than parallel, processes of suppression of an automatic reflexive saccade and programming and generation of a volitional saccade. In the ipisilateral antisaccade trials, the visual cue appeared on the side contralateral to the tDCS. If cathodal tDCS over FEF were to impair the suppression of reflexive saccade to the contralateral visual cue, it would take longer to suppress the reflexive saccade to the contralateral visual cue and thereby delay the programming of an ipisilateral saccade.

The finding of prolongation of ipisilateral antisaccade is consistent with previous TMS studies over FEF in the antisaccade task (Müri et al., 1991; Olk et al., 2006). In those studies, single pulse TMS was delivered over FEF either $50 \mathrm{~ms}$ before (Olk et al., 2006) or 50-90 ms after (Müri et al., 1991) the onset of a visual cue. In both cases, prolongation of the latency for ipisilateral saccades was found and no prolongation for contralateral antisaccades was found. These results suggest that both tDCS and TMS over FEF primarily interfere with suppression of reflexive saccades to the contralateral visual cue.

\section{CHANGES IN INHIBITION ERRORS AFTER tDCS}

A more direct way to test the idea that tDCS over FEF interferes with suppression of reflexive saccades is to examine the changes in the inhibition error rate after $\mathrm{tDCS}$. If the cathodal tDCS over FEF impaired the ability to suppress reflexive saccades, the number of error saccades would be expected to increase. However, our results did not show a significant increase in the error rate (Figure 4D). It is possible that our experiment was not sensitive enough to detect a subtle change, because the typical rate of inhibition errors was about $5 \%$ of trials in our study and our estimates of the error rate were based on 40 saccades per condition for each subject. Therefore, one error saccade comprised $2.5 \%$ of trials and a small variability in the error rates would be very difficult to detect in the current study.

On the other hand, we did detect a small change in the inhibition error rate with the anodal tDCS, which produced a significant change. It reduced the errors of making saccades to the visual cue presented contralateral to the stimulation electrode. This is again consistent with the idea that $\mathrm{tDCS}$ over FEF modulates the suppression of reflexive saccades. That is, our results can be interpreted as anodal tDCS facilitated the FEF function to suppress erroneous reflexive saccades to contralateral visual cues.

\section{LACK OF EFFECTS ON SACCADE AMPLITUDE AND VARIABILITY}

Although anodal tDCS shortened the contralateral saccade latency, we did not find a change in accuracy as measured by the landing point and variability, which would be expected if one considered a potential trade-off between saccade latency and accuracy (Schall, 1995). A similar result was found in a TMS experiment in which TMS had an effect on latency but not accuracy (Priori et al., 1993). The antisaccade task is known to engage the FEF more than a prosaccade task (Connolly et al., 2002) and therefore it was expected that $\mathrm{TDCS}$ over FEF might produce a more pronounced effect on the saccade amplitude and variability. However, this was not observed. One possible reason for the lack of effects on saccade size is due to the fact that saccade target positions were always indicated by the hollow squares making the programming of saccade amplitude and directions robust to manipulations by tDCS.

\section{COMPARISON OF tDCS EFFECTS BETWEEN THE PROSACCADE AND ANTISACCADE TASKS}

So far, we have explained the effects of tDCS on prosaccades and antisaccade invoking a different mechanism for each. To explain the effect of cathodal tDCS in the antisaccade task, we assumed that cathodal tDCS impairs the suppression of contralateral reflexive saccade, and for the facilitation of prosaccades by anodal tDCS, we explained that prosaccade latency was shortened by enhanced activity level of FEF. These two explanations may appear to conflict with each other. The interpretation of the FEF activity as the efficiency of suppressing contralateral saccade contradicts the results of the prosaccade experiment. Conversely, if we interpret the FEF activity as the speed of saccadic response as we did for explaining the results of the prosaccade experiment, the results of the antisaccade experiment cannot be explained. For instance, the suppression hypothesis predicts that cathodal tDCS would shorten contralateral prosaccades by reducing the suppression of saccade generation. However, our results showed no effect, or if anything an opposite effect.

These apparent conflicts can be reconciled if we consider (1) that the effect of tDCS is dependent on the activity level during the task and (2) that FEF consists of both saccade execution neurons and saccade suppression neurons. In support of the first 
point, an earlier study of tDCS showed that when tDCS was delivered over the motor cortex while subjects were engaged in a mental task, deactivating the motor cortex, subsequent effects of the tDCS on motor-evoked potentials were much reduced compared with delivery of tDCS during a passive condition (Antal et al., 2007). This suggests that neurons that are active during tDCS are more susceptible to the modulation by tDCS.

As for the second point, it is known that subset of FEF neurons with foveal receptive fields serve for suppressing saccade execution (Segraves and Goldberg, 1987) in a manner analogous to the rostral superior colliculus (Munoz and Wurtz, 1992, 1993). Moreover microstimulation of those neurons in FEF prolongs saccade latency in macaque monkeys (Burman and Bruce, 1997). Given the task dependency of tDCS, it is plausible that different subtypes of FEF neurons are affected between the prosaccade and antisaccade tasks. Specifically, in the prosaccade task, there is no task demand to suppress a reflexive saccade and therefore saccade execution neurons would be more active. On the other hand, saccade suppression neurons would be much more active during the antisaccade task. Therefore, it can be inferred that effects of tDCS was stronger for the saccade execution neurons in the prosaccade task, whereas it was stronger for the saccade suppression neurons in the antisaccade task. This framework provides explanations for the main two positive findings, namely, the effect of cathodal tDCS on antisaccades and the effect of anodal tDCS on prosaccades.

However, there remain two predictions made by this framework that are not measured in the present data. First, cathodal tDCS should have lengthened contralateral prosaccades. Second, anodal tDCS should have shortened ipsilateral antisaccade. As for the lack of these effects, it is possible that the effect size was too small to detect with our experimental procedures. In the unilateral cathodal tDCS condition, the prosaccade latency was slightly $(\sim 3 \mathrm{~ms})$ longer for the contralateral than ipsilateral saccades (Figure 2F). Since reflexive prosaccades are not heavily dependent on FEF (Pierrot-Deseilligny et al., 1991; Rivaud et al., 1994), suppression of FEF by cathodal tDCS may not be effective for increasing the latency. In the unilateral anodal tDCS condition, ipsilateral antisaccades were slightly $(\sim 3 \mathrm{~ms})$ longer, which is opposite to the prediction. However, the $3 \mathrm{~ms}$ differences were too small to reliably capture with the sampling rate of the eye tracker used in the present study $(250 \mathrm{~Hz})$; one frame corresponded to $4 \mathrm{~ms}$, While the predictions mentioned here are not confirmed in our current experiments, those effects may be revealed with stronger stimulation protocols which may lead to a larger effect size.

\section{PRACTICE EFFECT}

One of the weaknesses of our present study is the lack of a sham condition, which introduces ambiguity as to whether latency change over time comes from effects of tDCS or practice over trials. While the unilateral tDCS conditions were used as a way to introduce a no stimulation condition, we cannot rule out the possibility that the FEF on the unstimulated side was modulated by tDCS over the contralateral FEF via interhemispheric connectivity between homologous regions (e.g., Suppa et al., 2008). In addition, there is a limitation in the comparison of the results of the unilateral
tDCS conditions with the results of the bilateral tDCS experiment. Effects of tDCS tend to become weaker when extracephalic reference electrodes are used as in our unilateral tDCS conditions (Moliadze et al., 2010).

\section{HEMISPHERIC ASYMMETRY OF FEF IN HUMANS}

Hemispheric differences in the functions of human FEF have been reported in several aspects cognitive functions such as top-down attention to early visual areas (Silvanto et al., 2006; Ruff et al., 2009), conjunction search (Muggleton et al., 2003), and motor selection (Bardi et al., 2012). For example, the TMS over the left and right FEF exhibits different effects on visual areas. TMS over the left FEF increased the sensitivity of left MT/V5 (i.e., reduction of threshold for TMS-induced phosphenes in MT/V5), whereas the effect of TMS over the right FEF was observed in both left and right MT/V5 (Silvanto et al., 2006). A concurrent TMS-fMRI study showed that TMS over the left and right FEF both deactivates early visual areas corresponding to the central visual field, whereas activation of early visual areas representing visual periphery was specific to TMS over the right FEF (Ruff et al., 2009). These findings are compatible with the suggestion from clinical observations that the right FEF plays a more general attentional role covering both visual fields, whereas the role of the left FEF is more limited and restricted to contralateral visual field (Mesulam, 1981). Such hemispheric differences could potentially have added variability to our data. Further tDCS studies will be informative to further determine hemispheric differences in the function of the FEF.

\section{COMPARISON OF tDCS AND TMS}

The effects of anodal tDCS on prosaccade latency both in the bilateral and unilateral stimulation and the effects of cathodal tDCS in the antisaccade task both suggest that the induced electric current reached the FEF as intended. Further, as we have discussed, the effects of cathodal tDCS were by and large consistent with previous offline TMS studies on FEF both in the antisaccade tasks (Müri et al., 1991; Olk et al., 2006), again suggesting that the source of the effects is disruption of FEF.

One disadvantage of tDCS is that compared to TMS, the stimulation spreads to larger cortical regions due to the volume conduction (see, Wagner et al., 2006, 2007a,b) and it is more difficult to ensure the intended target cortical area is stimulated. A study of tDCS over the motor cortex showed that effectiveness of tDCS depends the position of the reference electrode (Nitsche and Paulus, 2000) because the direction of current flow with respect to the cortical surface is critical for excitability change by tDCS (Landau et al., 1964). The optimal electrode montage for FEF stimulation therefore remains to be determined. To obtain larger effects in future studies, it is important to consider the possibility that different electrode montages may induce greater effects.

One of the advantages of tDCS over TMS for interfering with FEF functions is that tDCS does not produce uncomfortable twitches or eye blinks. Also, the auditory and tactile sensations produced by a TMS pulse briefly suppress the generation of microsaccades for a few hundred milliseconds (Kanai et al., 2008). Such an artifact would potentially be a serious concern in oculomotor studies. 


\section{CONCLUSION}

In summary, our present study shows that tDCS can be used to modulate activity of FEF over a time course of up to $30 \mathrm{~min}$. It opens the possibility of applying tDCS for studying functions of FEF and for enhancing oculomotor and attentional performance in the future.

\section{REFERENCES}

Antal, A., Kincses, T. Z., Nitsche, M. A., and Paulus, W. (2003). Manipulation of phosphene thresholds by transcranial direct current stimulation in man. Exp. Brain Res. 150, 375-378.

Antal, A., Nitsche, M. A., Kruse, W., Kincses, T. Z., Hoffmann, K.-P., and Paulus, W. (2004). Direct current stimulation over V5 enhances visuomotor coordination by improving motion perception in humans. J. Cogn. Neurosci. 16, 521-527.

Antal, A., Nitsche, M. A., and Paulus, W. (2006). Transcranial direct current stimulation and the visual cortex. Brain Res. Bull. 68, 459-463.

Antal, A., Terney, D., Poreisz, C., and Paulus, W. (2007). Towards unravelling task-related modulations of neuroplastic changes induced in the human motor cortex. Eur. J. Neurosci. 26, 2687-2691.

Bardi, L., Kanai, R., Malpelli, D., and Walsh, V. (2012). TMS of the FEF interferes with spatial conflict. $J$. Cogn. Neurosci. 24, 1305-1313.

Baudewig, J., Nitsche, M. A., Paulus, W., and Frahm, J. (2001). Regional modulation of BOLD MRI responses to human sensorimotor activation by transcranial direct current stimulation. Magn. Reson. Med. 45, 196-201.

Bindman, L. J., Lippold, O. C. J., and Redfearn, J. W. T. (1962). Longlasting changes in the level of the electrical activity of the cerebral cortex produced by polarizing currents. Nature 196, 584-585.

Burman, D. D., and Bruce, C. J. (1997). Suppression of task-related saccades by electrical stimulation in the primate's frontal eye field. J. Neurophysiol. 77, 2252-2267.

Carpenter, R. H., and Williams, M. L. (1995). Neural computation of log likelihood in control of saccadic eye movements. Nature 377, 59-62.

Connolly, J. D., Goodale, M. A., Menon, R. S., and Munoz, D. P. (2002). Human fMRI evidence for the neural correlates of preparatory set. Nat. Neurosci. 5, 1345-1352.

Cornelissen, F. W., Kimmig, H., Schira, M., Rutschmann, R. M., Maguire, R. P., Broerse, A., den Boer, J. A., and Greenlee, M. W. (2002). Eventrelated fMRI responses in the human frontal eye fields in a randomized pro- and antisaccade task. Exp. Brain Res. 145, 270-274.
Creutzfeldt, O. D., Fromm, G. H., and Kapp, H. (1962). Influence of transcortical d-c currents on cortical neuronal activity. Exp. Neurol. 5, 436-452.

Dias, E. C., and Segraves, M. A. (1999). Muscimol-induced inactivation of monkey frontal eye field: effects on visually and memoryguided saccades. J. Neurophysiol. 81, 2191-2214.

Ettinger, U., ffytche, D. H., Kumari, V., Kathmann, N., Reuter, B., Zelaya, F., and Williams, S. C. R. (2008). Decomposing the neural correlates of antisaccade eye movements using event-related fMRI. Cereb. Cortex 18, 1148-1159.

Everling, S., and Fischer, B. (1998). The antisaccade: a review of basic research and clinical studies. Neuropsychologia 36, 885-899.

Fregni, F., and Pascual-Leone, A. (2007). Technology insight: noninvasive brain stimulation in neurology-perspectives on the therapeutic potential of rTMS and tDCS. Nat. Clin. Pract. Neurol. 3, 383-393.

Gaymard, B., Ploner, C. J., RivaudPéchoux, S., and Pierrot-Deseillign, C. (1999). The frontal eye field is involved in spatial short-term memory not in reflexive saccade inhibition. Exp. Brain Res. 129, 288-301.

Guitton, D., Buchtel, H. A., and Douglas, R. M. (1985). Frontal lobe lesions in man cause difficulties in suppressing reflexive glances and in generating goal-directed saccades. Exp. Brain Res. 58, 455-572.

Hallet, P. E. (1978). Primary and secondary saccades to goals defined by instructions. Vision Res. 18, 1279-1296.

Hanes, D. P., and Schall, J. D. (1996). Neural control of voluntary movement initiation. Science 274, 427-430.

Hunt, A., Olk, B., von Mühlenen, A., and Kingstone, A. (2004). Integration of competing saccade programs. Brain Res. Cogn. Brain Res.19, 206-208.

Juan, C.-H., Muggleton, N. G., Tzeng, O. J. L., Hung, D. L., Cowey, A., and Walsh, V. (2008). Segregation of visual selection and saccades in human frontal eye fields. Cereb. Cortex 18, 2410-2415.

Kanai, R., Muggleton, N. G., and Walsh, V. (2008). TMS over the intraparietal sulcus induces perceptual fading. $J$. Neurophysiol. 100, 3343-3350.
Landau, W. M., Bishop, G. H., and Clare, M. H. (1964). Analysis of the form and distribution of evoked cortical potentials under the influence of polarizing currents. J. Neurophysiol. 27, 788-813.

Matsuda, T., Matsuura, M., Ohkubo, T., Ohkubo, H., Matsushima, E., Inoue, K., Taira, M., and Kojima, T. (2004). Functional MRI mapping of brain activation during visually guided saccades and antisaccades: cortical and subcortical networks. Psychiatry Res. 131, 147-155.

McDowell, J. E., Brown, G. G., Paulus, M., Martinez, A., Stewart, S. E. Dubowitz, D. J., and Braff, D. L. (2002). Neural correlates of refixation saccades and antisaccades in normal and schizophrenia subjects. Biol. Psychiatry 51, 216-223.

Mesulam, M. M. (1981). A cortical network for directed attention and unilateral neglect. Ann. Neurol. 10, 309-325.

Mohler, C. W., Goldberg, M. E., and Wurtz, R. H. (1973). Visual receptive fields of frontal eye field neurons. Brain Res. 61, 385-389.

Moliadze, V., Antal, A., and Paulus, W. (2010). Electrode-distance dependent after-effects of transcranial direct and random-noise stimulation with extracephalic reference electrodes. Clin. Neurophysiol. 121, 2165-2171.

Muggleton, N. G., Juan, C.-H., Cowey, A., and Walsh, V. (2003). Human frontal eye fields and visual search. J. Neurophysiol. 89, 3340-3343.

Munoz, D. P., and Everling, S. (2004). Look away: the anti-saccade task and the voluntary control of eye movement. Nat. Rev. Neurosci. 5, 218-228.

Munoz, D. P., and Wurtz, R. H. (1992). Role of the rostral superior colliculus in active visual fixation and execution of express saccades. J. Neurophysiol. 67, 1000-1002.

Munoz, D. P., and Wurtz, R. H. (1993). Fixation cells in monkey superior colliculus. I. Characteristics of cell discharge. J. Neurophysiol. 70, 559-575.

Müri, R. M., Heid, O., Nirkko, A. C., Ozdoba, C., Felblinger, J., Schroth, G., and Hess, C. W. (1998). Functional organisation of saccades and antisaccades in the frontal lobe in humans: a study with echo planar functional magnetic resonance imaging. J. Neurol. Neurosurg. Psychiatr. 65, 374-377.

Müri, R. M., Hess, C. W., and Meienberg, O. (1991). Transcranial stimulation of the human frontal eye field by magnetic pulses. Exp. Brain Res. 86, 219-223.

Nitsche, M. A., Cohen, L. G., Wassermann, E. M., Priori, A., Lang, N., Antal, A., Paulus, W., Hummel, F., Boggio, P. S., Fregni, F., and PascualLeone, A. (2008). Transcranial direct current stimulation: state of the art 2008. Brain Stimul. 1, 206-223.

Nitsche, M. A., and Paulus, W. (2000). Excitability changes induced in the human motor cortex by weak transcranial direct current stimulation. J. Physiol. 527, 633-639.

Nitsche, M. A., and Paulus, W. (2001). Sustained excitability elevations induced by transcranial DC motor cortex stimulation in humans. Neurology 57, 1899-1901.

Nuding, U., Kalla, R., Muggleton, N. G., Büttner, U., Walsh, V., and Glasauer, S. (2009). TMS evidence for smooth pursuit gain control by the frontal eye fields. Cereb. Cortex 19, 1144-1150.

Nyffeler, T., Wurts, P., Luscher, H. R., Hess, C. W., Senn, W., Pflugshaupt, T., von Warburg, R., Luthi, M., and Müri, R. M. (2006a). Extending lifetime of plastic changes in the human brain. Eur. J. Neurosci. 24, 2961-2966.

Nyffeler, T., Wurts, P., Luscher, H. R., Hess, C. W., Senn, W., Pflugshaupt, T., von Warburg, R., Luthi, M., and Müri, R. M. (2006b). Repetitive TMS over the human oculomotor cortex: comparison of $1-\mathrm{Hz}$ and theta burst stimulation. Neurosci. Lett. 409, 57-60.

O’Driscoll, G. A., Alpert, N. M. Matthysse, S. W., Levy, D. L., Rauch, S. L., and Holzman, P. S. (1995). Functional neuroanatomy of antisaccade eye movements investigated with positron emission tomography. Proc. Natl. Acad. Sci. U.S.A. 92, 925-929.

Olk, B., Chang, E., Kingstone, A., and Ro, T. (2006). Modulation of antisaccades by transcranial magnetic stimulation of the human frontal eye field. Cereb. Cortex 16, 76-82.

Paus, T. (1996). Location and function of the human frontal eye fields. Neuropsychologia 34, 475-483. 
Pierrot-Deseilligny, C., Rivaud, S., Gaymard, B., and Agid, Y. (1991). Cortical control of reflexive visuallyguided saccades. Brain 114(Pt 3), 1473-1485.

Priori, A., Berardelli, A., Rona, S., Accornero, N., and Manfredi, M. (1998). Polarization of the human motor cortex through the scalp. Neuroreport 9, 2257-2260.

Priori, A., Bertolasi, L., Rothwell, J. C., Day, B. L., and Marsden, C. D. (1993). Some saccadic eye movements can be delayed by transcranial magnetic stimulation of the cerebral cortex in man. Brain 116, 355-367.

Reddi, A. J., Asrress, K. N., and Carpenter, R. H. S. (2003). Accuracy, information and response time in a saccadic decision task. J. Neurophysiol. 90, 3538-3546.

Rivaud, S., Müri, R., Gaymard, B., Vermersch, A. I., and Pierrot-Deseilligny, C. (1994). Eye movement disorders after frontal eye field lesions in humans. Exp. Brain Res. 102, 110-120.

Robinson, D. L., and Fuchs, A. F. (1969). Eye movements evoked by stimulation of frontal eye fields. J. Neurophysiol. 32, 637-648.

Ruff, C. C., Blankenburg, F., Bjoertomt, O., Bestmann, S., Weiskopf, N., and
Driver, J. (2009). Hemispheric differences in frontal and parietal influences on human occipital cortex: direct confirmation with concurrent TMS-fMRI. J. Cogn. Neurosci. 21, 1146-1161.

Sack, A. T., Cohen Kadosh, R., Schuhmann, T., Moerel, M., Walsh, V., and Goebel, R. (2009). Optimizing functional accuracy of TMS in cognitive studies: a comparison of methods. J. Cogn. Neurosci. 21, 207-221.

Schall, J. D. (1995). Neural basis of saccade target selection. Rev. Neurosci. 6, 63-85.

Schall, J. D., and Thompson, K. G. (1999). Neural selection and control of visually guided eye movements. Annu. Rev. Neurosci. 22, 241-259.

Segraves, M. A., and Goldberg, M. E. (1987). Functional properties of corticotectal neurons in the monkey's frontal eye field. J. Neurophysiol. 58, 1387-1419.

Serences, J. T., and Yantis, S. (2007). Spatially selective representations of voluntary and stimulus-driven attentional priority in human occipital, parietal and frontal cortex. Cereb. Cortex 17, 284-293.

Silvanto, J., Lavie, N., and Walsh, V. (2006). Stimulation of human frontal eye fields modulates sensitivity of extrastriate visual cortex. $J$. Neurophysiol. 96, 941-945.

Sommer, M. A., and Tehovnik, E. J. (1997). Reversible inactivation of macaque frontal eye field. Exp. Brain Res. 116, 229-249.

Suppa, A., Ortu, E., Zafar, N., Deriu, F., Paulus, W., Berardelli, A., and Rothwell, J. C. (2008). Theta burst stimulation induces after-effects on contralateral primary motor cortex excitability in humans. J. Physiol. 586, 4489-4500.

Wagner, T., Fregni, F., Eden, U., Ramos-Estebanez, C, Grodzinsky, A., Zahn, M., and Pascual-Leone, A. (2006). Transcranial magnetic stimulation and stroke: a computerbased human model study. Neuroimage 30, 857-870.

Wagner, T., Fregni, F., Fecteau, S., Grodzinsky, A., Zahn, M., and Pascual-Leone, A. (2007a). Transcranial direct current stimulation: a computer-based human model study. Neuroimage 35, 1113-1124.

Wagner, T., Valero-Cabre, A., and Pascual-Leone, A. (2007b). Noninvasive human brain stimulation. Annu. Rev. Biomed. Eng. 9, 527-565.

White, J. M., Sparks, D. L., and Stanford, T. R. (1994). Saccades to remembered target locations: analysis of systematic and variable errors. Vision Res. 34, 79-92.

Wurtz, R. H., and Mohler, C. W. (1976). Enhancement of visual response in monkey striate cortex and frontal eye fields. J. Neurophysiol. 39, 666-722.

Conflict of Interest Statement: The authors declare that the research was conducted in the absence of any commercial or financial relationships that could be construed as a potential conflict of interest.

Received: 08 January 2012; accepted: 22 April 2012; published online: 10 May 2012.

Citation: Kanai $R$, Muggleton $N$ and Walsh V (2012) Transcranial direct current stimulation of the frontal eye fields during pro- and antisaccade tasks. Front. Psychiatry 3:45. doi: 10.3389/fpsyt.2012.00045

This article was submitted to Frontiers in Neuropsychiatric Imaging and Stimulation, a specialty of Frontiers in Psychiatry. Copyright (C) 2012 Kanai, Muggleton and Walsh. This is an open-access article distributed under the terms of the Creative Commons Attribution Non Commercial License, which permits noncommercial use, distribution, and reproduction in other forums, provided the original authors and source are credited. 